\title{
Training through i-Maths to potentiate specific cognitive domain performance scores in young kids
}

Roohollah Zahediannasb ${ }^{1,2,3, \Delta}$, Maryam Hosseini1,2,3,4, Seyedeh Saeedeh Yahyavi1,2,3, AliMohammad Kamali1,2,3, Mahshid Tahamtan ${ }^{1,2,3}$, Mohammad-Mojtaba Kamelmanesh ${ }^{4,5}$, Mohammad Nami1,2,3,6*

1Neuroscience Laboratory (Brain, Cognition and Behavior), Department of Neuroscience, School of Advanced Medical Sciences and Technologies, Shiraz University of Medical Sciences, Shiraz, Iran

2Department of Neuroscience, School of Advanced Medical Sciences and Technologies, Shiraz University of Medical Sciences, Shiraz, Iran

3DANA Brain Health Institute, Iranian Neuroscience Society, Fars Chapter, Shiraz, Iran

${ }^{4}$ ATRAK, Educational and Scientific Institute, Tehran, Iran

5Islamic Azad University, Fars Branch, Shiraz, Iran

${ }^{6}$ Academy of Health, Senses Cultural, Sacramento, CA, USA

$\Delta:$ These authors have made equal contribution to this report.

*Corresponding author: Mohammad Nami MD, PhD, Department of Neuroscience, School of Advanced Medical Sciences and Technologies, Shiraz University of Medical Sciences, Shiraz, Iran. torabinami@sums.ac.ir

\begin{abstract}
Background and Objectives: i-Maths is a new math educational program based on conceptualizing mathematical concepts for children aged 3-7 years. The early years of life are considered crucial for cognitive construct development and learning where the figure-sense and mathematical concepts start encoding. Studies show that spatial working memory plays an important role in learning mathematics upon childhood. This study was an attempt to investigate whether a formal course of $i$ Maths training would potentially improve cognitive capacities such as short-term memory in children.

Material and Methods: 20 kids ( 7 girls and 12 boys, aged 5 to 7 years) were randomly selected and enrolled from an i-Maths training institute in Shiraz and stratified in two groups i.e. experienced and novice ( $n=10$ in each group). In both groups, the participants' cognitive performance was measured and profiled using the Cambridge Neuropsychological Test Automated Battery (CANTAB). In addition, their hemodynamic response from the frontopolar brain region (FP1) was measures using the hemoencephalography (HEG) setup upon CANTAB task performance.

Results: Subjects in both groups showed no significant difference in motor screening task (MOT) scores. Meanwhile, in the delayed matching to sample (DMS), there was significant difference in task scores in the experienced- as compared to the novice-group. Also the results of paired associates learning (PAL) shows significant difference in total number of errors (6 shapes adjusted) in the experienced- vs. novice-group. Nevertheless, the changes were not significant in terms of total errors (adjusted). Moreover, there was no significant change in HEG response in two groups.

Conclusion: It seems that learning mathematical concepts through i-Maths leaves favorable outcomes in distinct cognitive performance domains in young kids. Further research would need to unravel neurodynamics involved in such improvements.
\end{abstract}

Keywords: i-Maths, Cognitive performance, Hemodynamic response, CANTAB, Young kids 


\section{Introduction}

Mathematics consists of a set of ideas and activities including measurement, comparison, calculating, reasoning and so on (1). So far, several definitions have been proposed for mathematics that correspond to the proposer of each definition and the benefits that they can derive from this science (2). For example, one can view mathematics as a tool that is used in various sciences (for example, in sciences, engineering, etc.). It can also be considered as a discipline which can be studied or taught, or can be used as a way of thinking (2). In recent years, mathematics and its applications have attracted a lot of attention.

Mathematics is one of the main pillars of modern economics (3). That is why the general interest in learning mathematics, especially by children and in pre-school ages, has increased dramatically. The prime of childhood is a very important opportunity for children to learn, especially in the field of mathematics (4). In the early years of life, children can learn mathematics and increase their skills as well as their interest in math learning (4).

The significance of early childhood education can be studied from two perspectives, i.e. their sensitivity and susceptibility to receive influence in the educational environments, and the durability and depth of their learning in this period of life (5). In other words, early learning provides a good basis for the later experiences of children, and what is learned in the early years of life and in preschool years is more stable and not easily eliminated.

That said, it appears that what young children learn early on, can predict their educational achievements in the future, because this early experience in math learning will affect their future learning of math and even other fields. This elementary education plays an important role in the course of children development, as researches have shown that many learning disorders can be prevented with primary education (6).

Studies show that working memory plays an important role in early learning of mathematics, and that children who show a weaker performance in this function are likely to be at a higher risk of learning disabilities in the future (7). The neural circuits related to spatial working memory in the brain play a key role in the early learning of mathematics (8). It has been shown that about $7 \%$ of children with learning disabilities have some degrees of cognitive impairment and psychiatric disorders (9). Therefore, it seems that mathematics is one of the main interdigited domains of cognition. One way to familiarize children with math concepts is implementation of educational programs by related institutions that teach math skills, problem solving, as well as concepts such as number, shape, time, measurement, data analysis, and problem solving in ways that motivate young children.

A study by Friso-van den Bos in 2013 found that individuals who have a better performance in mathematics have also a better performance in terms of working memory, including inhibition, shifting, and updating (10). In a study in 2006 on children aged 11-12 years, Helen L. et al. showed that the better performance of these children in working memory, especially its inhibition, is related to mathematics, and those who do better in mathematics tend to outperform in working memory (11).

I-Maths is a new math educational program for children aged 3-7 years. The main goals of this training program are to strengthen skills such as observing, thinking, and reasoning (www.imath.sg). 
I-Maths is a maths program for young children which is designed in accordance with the pattern of how children learn to understand mathematical concepts. Through mathematics, children learn to understand their world in terms of numbers and shapes. Through puzzles, activities, games, tangrams - math can actually become a child's play. They learn to connect ideas to reason and to think logically. Besides Iran, i-Maths is successfully running in China, Singapore, Malaysia Indonesia and India.

The question whether math education to children ages 5-7 using the i-Maths can improve cognitive skills such as reasoning, concentration, and short-term memory in these children compared to children who have not undergone such an educational pathway prompted us to design and run the present investigation.

\section{Materials and Methods \\ 2.1. Subjects}

A total of 20 Kids ( 7 girls and 12 boys, age 5 to 7 years) were randomly selected and enrolled from an i-Maths training institute in Shiraz. They were divided into two age- and sexmatched groups including the experienced and novice groups ( $n=10$ each group). In the experienced group, the children were at the highest course level of i-Math training, whereas in the novice group they have either had limited course of i-Maths training or registered recently. The inclusion criteria included 1-right-handedness, 2-acquiring at least moderate intelligence score evaluated by the Raven's Progressive Matrices, 3- having no mental disorders, chronic disease, history of traumatic brain injury, attention deficit hyperactivity disorder (ADHD) or physical defects deterring the testing procedure.

The Conner's Teacher Rating Scale (CTRS) was also performed to assesses psychiatry problems in children specially behaviors related to ADHD (12). This scale is one of the valid questionnaires commonly used by researchers and practitioners. This scale has been tested for validity and reliability in various cultural and ethnic backgrounds. The present study employed the Persian version of CTRS whereby only those receiving score less than 57 were enrolled.

Parents of all enrolled children were debriefed about the procedure and that they can withdraw from the procedure wherever in the study course on their own will. As such, they provided informed consent and the study protocol was evaluated and approved by the institutional ethical review board at Shiraz University of Medical Sciences.

\subsection{Cognitive assessment}

The Cambridge Neuropsychological Test Automated Battery (CANTAB) was used to assess domain-specific cognitive performance scores between groups. The employed licensed CANTAB battery was a computer platform (CANTAB Research Suite 6.0.37, eclipse-6.0.xrev.31911) comprising 25 tests to examine various cognitive functions including visual, verbal and episodic memory, attention, executive function, reaction time, information processing, decision making and response control. We used three CANTAB subtests with non-verbal patterns including the motor screening task (MOT), delayed matching to sample (DMS), and paired associates learning (PAL) in a silent room without distractions (https://www.cambridgecognition.com/cantab/). 
Frist, the subjects were asked to perform the MOT. MOT was used as a training tool at the beginning of the testing session where subjects should have touched a color cross as soon as it appears randomly anywhere on the screen. This test is used to measure speed, ideomotor accuracy and number of errors which represent sensorimotor skills. As such, children who failed to perform MOT were excluded the study.

The PAL test measures substrates of visual memory and learning. In this test, several shapes appear on different parts of the screen in 5 stages and then hide. At each step there are one, two, three, six and eight shapes respectively and the participant must touch the location of each shape on the screen when they appear on the screen center.

In DMS, both simultaneous visual matching ability and short-term visual recognition memory are assessed.In this test, the participant is asked to match the shapes shown on the screen with the shapes shown in the earlier trial.

\subsection{Hemoencephalography}

Hemoencephalography is a new neurofeedback technique (Peanut nIR HEG kit) to measure cerebral blood flow in prefrontal cortex specially frontopolar by passive infrared light emission and uptake. Using this technique, the neural activity is measured indirectly following an increased in the metabolic activity and hence hemodynamic response over a cerebral cortical region when specific cognitive task is performed $(13,14)$.

In the present investigation we have measures the left frontopolar (FP1) HEG response upon resting state for 2 minutes and then upon CANTAB task performance in all subjects from the experienced and the novice groups.

\subsection{Statistical analysis}

Based on the normality of distribution and homogeneity of variance parametric and nonparametric statistical tests including the one-tailed $t$-test and Mann-Whitney were employed. The statistical analysis was mainly carried out using the one-tailed independent $t$ test. A p-value $<0.05$ was considered as statistically significant.

\section{Results}

Based on the Raven's intelligence test, the mean score of intelligence in the experienced and novice groups were $105 \pm 4$ and $100 \pm 3$, respectively. In addition, the Conner's questionnaire also confirmed the concordance with participants' inclusion criteria.

The MOT provides a general assessment of sensorimotor impairment or lack of understanding that limits the collection of valid information from the participant. Findings from this task confirmed that both groups were eligible in undergoing CANTAB tasks. In fact, the two groups do not significantly differ in terms of their task score. 
There was a significant difference between the two groups (experienced vs. novice) in the DMS percent correct $(p=0.005)$ and DMS percent correct (all delays) $(p=0.03)$ while the comparison did not reveal any significant difference in DMS percent correct (simultaneous) $(p=0.4)$. These data are illustrated

in Figure 1.

Delayed Matching to Sample test uses non-verbal patterns to evaluated both simultaneous visual matching ability and short-term visual recognition memory.

Visual memory and new learning capacity were evaluated using the Paired Associates Learning task with results showing significant difference in total errors (6 shapes adjusted) ( $p=0.036)$ in the experienced vs. novice group; while no significant difference has shown in terms of total errors (adjusted) $(p=0.2)$.

A comparative analysis of the hemodynamic response in the left frontopolar cortex did not show any significant difference between the two groups through the analysis of FP1 HEG response differences in optical density $(p=0.81)$. Meanwhile, based on paired sample $t$-test, PAL-concurrent HEG responses in both groups were different from those of the baseline $(p=0.001)$.

\section{Discussion}

This study was an attempt to compare the cognitive capacity in novice and experienced i-Maths trainees. Early and mid-childhood is an important and sensitive period of life for the development of motor, cognitive and social skills (15).

Therefore, the question whether specific training protocols such as i-Maths are of tangible values for cognitive development and learning skills appealed to our research group's attention. a

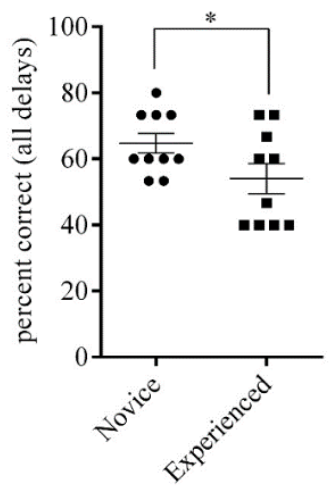

b

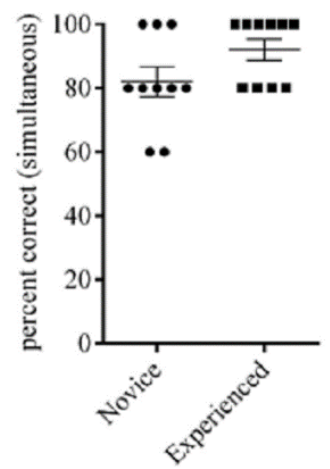

C

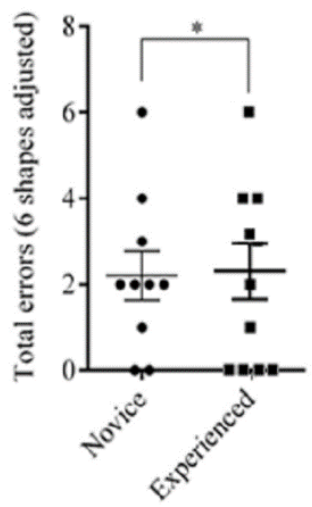

d
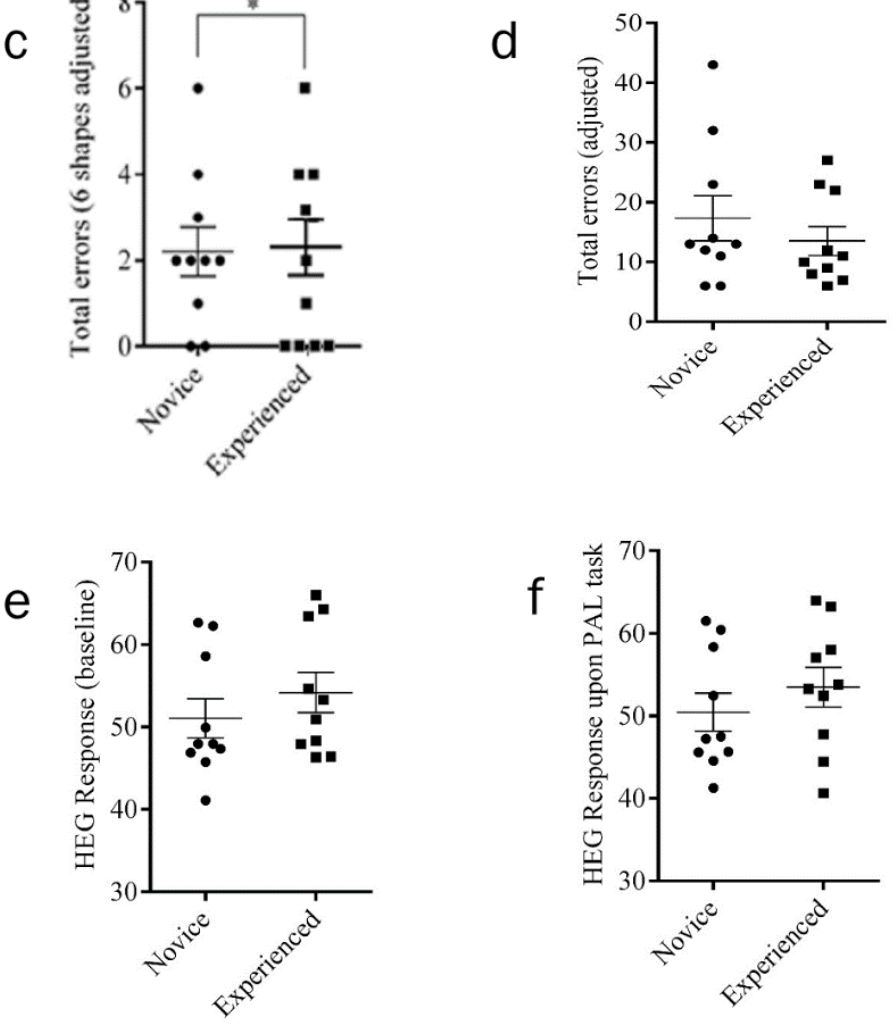

Figure 1. Scatter plots showing the performance scores of novice and experienced i-Maths trainees on CANTAB platform. Plots indicate a significant difference between the performance of novice and experienced subjects on DMS percent correct (all delays) and PAL total error (6 shape adjusted) (a,b,c) $(P<0.05)$. Meanwhile, there found to be no significant difference in PAL total error (adjusted) and HEG response at baseline and upon PAL (d,e,f) $(p>0.05)$. 
According to earlier reports, the use of multifaceted learning methods, combined with different senses and movement, leads to the best possible development of cognitive functions (16), which result in optimized hemodynamic response in the prefrontal, temporal and parietal regions (17) and regulated electrical neurodynamics in such cortical brain areas (18).

On the other hand, the fronto-temporo-parital (FTP) networks is associated with attention, memory, and executive functions (19), hence the reinforcement of such areas would be expected to evoke the development of cognitive functions in early and middle childhood. In the same vein, the multifaceted learning approached are shown to enhances this network $(19,20)$.

Cognitive functions such as attention, memory, and executive functions have close and bi-directional relationships with math. As such, reinforcing each may leave a reciprocal effects on the others hence predicting future successes (21).

I-Math is a multifaceted educational approach in which various concepts including maths are being taught through multi-sensory and motor involved tasks.

Findings from the present investigation provided preliminary insights into the impact of this multifaceted educational approach on the aptitude of cognitive functions. On the other hand, it was shown that when experienced i-Maths trainees were involved in PAL task their left frontopolar hemodynamic response was significantly increase compared to the resting state. The difference was not however found as significantly different between the two groups.

Given the mutual relation between math skills and development of specific cognitive domains particularly in the childhood period, the present study could partly prove its hypothesis and postulate the benefits of i-Maths on the aptitude of cognitive functions in young kids.

Though the present report suggested that i-Maths can potentially improve cognitive functions in our studied population, the mechanism through which such beneficial gains form over time and neurodynamics of the underlying neural plasticity, remain to be explained. Functional neuroimaging studies using the electrical, magnetic or optical modalities would help understanding the effects of i-Maths training on the functional connectivity within the FTP network.

\section{References}

1. Zaslavsky O, Shir K. Students' conceptions of a mathematical definition. Journal for Research in Mathematics Education. 2005:317-46.

2. Speer NM, King KD, Howell H. Definitions of mathematical knowledge for teaching: using these constructs in research on secondary and college mathematics teachers. Journal of Mathematics Teacher Education. 2015;18(2):105-22.

3. Hoy M, Livernois J, McKenna C, Rees R, Stengos T. Mathematics for economics: MIT press; 2011.

4. Clements DH. Mathematics in the preschool. Teaching children mathematics. 2001;7(5):270-.

5. Reys R, Lindquist M, Lindquist MM, Lambdin DV, Smith NL. Helping children learn mathematics: John Wiley \& Sons; 2014.

6. Melhuish EC, Sylva K, Sammons P, Siraj-Blatchford I, Taggart B, Phan MB, et al. Preschool influences on mathematics achievement. Science. 2008;321(5893):1161-2.

7. Passolunghi MC, Caviola S, De Agostini R, Perin C, Mammarella IC. Mathematics anxiety, working memory, and mathematics performance in secondary-school children. Frontiers in psychology. 2016;7:42. 
8. Peng P, Namkung J, Barnes M, Sun C. A meta-analysis of mathematics and working memory: Moderating effects of working memory domain, type of mathematics skill, and sample characteristics. Journal of Educational Psychology. 2016;108(4):455.

9. Fletcher JM, Lyon GR, Fuchs LS, Barnes MA. Learning disabilities: From identification to intervention: Guilford Publications; 2018.

10. Friso-Van Den Bos I, Van Der Ven SH, Kroesbergen EH, Van Luit JE. Working memory and mathematics in primary school children: A meta-analysis. Educational research review. 2013;10:29-44.

11. St Clair-Thompson HL, Gathercole SE. Executive functions and achievements in school: Shifting, updating, inhibition, and working memory. The quarterly journal of experimental psychology. 2006;59(4):745-

59.

12. Goyette $\mathrm{CH}$, Conners $\mathrm{CK}$, Ulrich RF. Normative data on revised Conners parent and teacher rating scales. J Abnorm Child Psychol. 1978;6(2):221-36.

13. Carmen JA. Passive infrared hemoencephalography: Four years and 100 migraines. Journal of Neurotherapy. 2005;8(3):23-51.

14. Pa TT. New developments in blood flow hemoencephalography: CRC Press; 2005.

15. Bidzan-Bluma I, Lipowska M. Physical activity and cognitive functioning of children: a systematic review. International journal of environmental research and public health. 2018;15(4):800.

16. Lim MH, Liu KP, Cheung GS, Kuo MC, Li R, Tong C-Y. Effectiveness of a multifaceted cognitive training programme for people with mild cognitive impairment: a one-group pre-and posttest design. Hong Kong Journal of Occupational Therapy. 2012;22(1):3-8.

17. Trangmar SJ, González-Alonso J. Heat, Hydration and the Human Brain, Heart and Skeletal Muscles. Sports Med. 2019;49(1):69-85.

18. Pan W-j, Thompson G, Magnuson M, Majeed W, Jaeger D, Keilholz S. Simultaneous FMRI and electrophysiology in the rodent brain. JoVE (Journal of Visualized Experiments). 2010(42):e1901.

19. Caruana N, Brock J, Woolgar A. A frontotemporoparietal network common to initiating and responding to joint attention bids. Neuroimage. 2015;108:34-46.

20. Feldman R. The adaptive human parental brain: implications for children's social development. Trends Neurosci. 2015;38(6):387-99.

21. Van der Ven SH, Kroesbergen EH, Boom J, Leseman PP. The development of executive functions and early mathematics: A dynamic relationship. Br J Educ Psychol. 2012;82(1):100-19. 